\title{
Laparoscopic sleeve gastrectomy in heart failure patients with left ventricular assist device
}

\begin{abstract}
Background: There is limited data in the literature evaluating outcomes of bariatric surgery in severely obese patients with left ventricular assist device (LVAD) as a bridge to make them acceptable candidates for heart transplantation. This study aims to assess the safety and effectiveness of laparoscopic sleeve gastrectomy (LSG) in patients with previously implanted LVAD at our institution. Methods: All the patients with end-stage heart failure (ESHF) and implanted LVAD who underwent LSG from2013 to January 2017 were studied. Results: Seven patients with end stage heart failure (ESHF) and implanted LVAD were included. The median age and median preoperative BMI were 39 years (range: 26-62) and $43.6 \mathrm{~kg} / \mathrm{m} 2$ (range 36.7-56.7), respectively. The median interval between LVAD implantation and LSG was 38 months (range 15-48). The median length of hospital stay was 9 days (rang: 6-23) out of which 4 patients had planned postoperative ICU admission. Thirty-day complications were noted in 5 patients ( 3 major and 2 minor) without any perioperative mortality. The median duration of follow-up was 24 months (range 2-30). At the last available follow-up, the median BMI, \%EWL, and \%TWL were $37 \mathrm{~kg} / \mathrm{m} 2,47 \%$, and $16 \%$, respectively. The median LVEF before LSG and at the last follow-up point (before heart transplant) was 19\% (range 15-20) and 22\% (range, 16-35), respectively. In addition, the median NYHA class improved from 3 to 2 after LSG. Three patients underwent successful heart transplantations. Conclusion: Patients with morbid obesity, ESHF, and implanted LVAD constitute a high-risk cohort. Our results with 7 patients and result from other studies (19 patients) suggested that bariatric surgery may be a reasonable option for LVAD patients with severe obesity. Bariatric surgery appears to provide significant weight loss in these patients and may improve candidacy for heart transplantation.
\end{abstract}

Keyword: Bariatric surgery; Heart failure; Heart transplantation; Left ventricular assist device; Sleeve gastrectomy; Weight loss; Perioperative complications 